\title{
Enfermedad Renal Crónica (ERC): Una guía de alimentos con alto contenido de fibra ${ }^{1}$
}

\author{
Wendy J. Dahl y Nancy J. Gal ${ }^{2}$
}

Vivir con la enfermedad renal crónica (ERC) presenta muchos desafíos, y la dieta es uno de ellos. Las personas con ERC pueden tener dificultades para consumir suficientes alimentos ricos en fibra al seguir las otras recomendaciones de dieta para la ERC. A medida que disminuye la función renal, a las personas con ERC se les recomienda limitar los alimentos con alto contenido de fósforo y potasio. Como algunos de los alimentos restringidos también son ricos en fibra, estas restricciones pueden resultar en una dieta baja en fibra.

No existen recomendaciones nacionales de ingesta de fibra específicas para personas con ERC (Krishnamurthy et al. 2012), pero generalmente se recomienda que las personas con ERC consuman una dieta más alta en fibra. Las recomendaciones de fibra para ERC pueden ser similares a las recomendaciones para individuos sanos: 21 a $25 \mathrm{~g}$ de fibra por día para mujeres y 30 a $38 \mathrm{~g}$ de fibra para hombres dependiendo en la edad (Trumbo et al. 2002). En promedio, las personas con ERC consumen solo alrededor de $15 \mathrm{~g}$ de fibra por día (Evenepoel and Meijers 2012). Sin embargo, es posible seguir las restricciones dietéticas para las etapas más avanzadas de la ERC y seguir consumiendo la cantidad recomendada de fibra. Esta guía discutirá los beneficios de la fibra en la salud y brindará algunos ejemplos de alimentos ricos en fibra que pueden ser buenas opciones para la ERC.

\section{Beneficios de fibra en la salud y la ERC}

La fibra se encuentra naturalmente en todos los alimentos de origen vegetal, incluyendo frutas, verduras, granos, nueces, semillas y legumbres. La fibra, cuando está aislada de las plantas, también puede ser agregada a los alimentos para crear alimentos con mayor contenido de fibra. El consumo adecuado de fibra mantiene un tracto digestivo saludable y reduce el riesgo de desarrollar enfermedades del corazón, diabetes tipo 2 y otras enfermedades crónicas (Dahl and Stewart 2015). También se recomiendan consumos más altos de fibra para el manejo de muchas enfermedades crónicas, ya que la fibra ayuda a reducir el colesterol y controlar el azúcar en la sangre. La fibra también puede ser beneficiosa para el control de la presión arterial (Aleixandre and Miguel 2016).

Consumir la cantidad recomendada de fibra puede tener una serie de beneficios para las personas con ERC. Un mayor consumo de fibra está relacionado con una mejor supervivencia (Krishnamurthy et al. 2012) al igual que los patrones dietéticos saludables que proporcionan fibra de frutas, verduras, legumbres y granos integrales (Kelly et al. 2016).

Consumo adecuado de fibra, en particular la fibra insoluble, puede ser útil para promover evacuación intestinal y prevenir el estreñimiento en personas con ERC (Salmean, Zello and Dahl 2013). La diabetes es la causa principal de

1. Este documento, FSHN18-6, es uno de una serie de publicaciones del Food Science and Human Nutrition, UF/IFAS Extensión. Fecha de primera publicación abril 2018. Visite nuestro sitio web EDIS en <http://edis.ifas.ufl.edu>.

2. Wendy J. Dahl, associate professor, Department of Food Science and Human Nutrition; and Nancy J. Gal, Extension agent IV, Food and Consumer Sciences, UF/IFAS Extension Marion County; UF/IFAS Extension, Gainesville, FL 32611.

The Institute of Food and Agricultural Sciences (IFAS) is an Equal Opportunity Institution authorized to provide research, educational information and other services only to individuals and institutions that function with non-discrimination with respect to race, creed, color, religion, age, disability, sex, sexual orientation, marital status, national origin, political opinions or affiliations. For more information on obtaining other UF/IFAS Extension publications, contact your county's UF/IFAS Extension office. 
insuficiencia renal. El consumo de alimentos que contienen fibra puede ayudar a controlar la glucosa en la sangre (Post et al. 2012), y así retrasar el progreso de la enfermedad (Shurraw et al. 2011; Lee et al. 2013). El aumento de riesgo de enfermedades cardiovasculares que acompaña a la ERC puede ser reducido con fibra ya que la fibra ayuda a reducir el colesterol y la inflamación (Dahl et al. 2016).

\section{Aumentando la fibra con la ERC}

En las primeras etapas de la ERC, cuando por lo general no hay restricciones de potasio, se recomienda consumir una variedad de alimentos que proporcionen fibra, como granos integrales, legumbres, frutas, verduras, nueces y semillas.

Muchos alimentos integrales y leguminosas son buenas fuentes de fibra, pero tradicionalmente han sido limitados en la dieta de la ERC debido a su contenido de fósforo.

Aunque los alimentos vegetales, como los granos integrales, contienen una cantidad significativa de fósforo, son buenas opciones de alimentos saludables para aquellos con ERC. Esto se debe a que solo una fracción del fósforo en los granos integrales se absorbe en el cuerpo (Williams, Ronco and Kotanko 2013). La Tabla 1 proporciona una lista de alimentos integrales.

Los alimentos con fibra añadida pueden ayudar a cumplir con las recomendaciones de fibra y pueden ser beneficiosos para la salud (Chiavaroli et al. 2014). La Tabla 2 brinda algunos ejemplos de alimentos a base de granos con fibra añadida. Para obtener más información sobre alimentos con fibra añadida, consulte http://edis.ifas.ufl.edu/pdffiles/ FS/FS23500.pdf.

Las frutas y verduras también son fuentes importantes de fibra. Las legumbres, como los frijoles, los guisantes y las lentejas, son especialmente ricas en fibra, proporcionando hasta $8 \mathrm{~g}$ de fibra en $1 / 2$ taza. Sin embargo, algunas personas con ERC tienen que restringir las legumbres y algunas frutas y verduras debido a su contenido de potasio. Para aquellas personas con ERC a quienes se les ha aconsejado restringir el potasio, hay frutas y vegetales bajas en potasio de las cuáles pueden elegir. Los ejemplos se enumeran en la Tabla 3.

Se recomienda que las personas con ERC consulten con un nutricionista dietista registrado (RDN), preferiblemente uno que se especialice en enfermedades renales, antes de cambiar su dieta. Un dietista puede ayudar a elegir las fuentes de fibra óptimas para cada etapa de la ERC.

\section{Material Suplementario}

Para más información sobre el contenido de fósforo de ciertos alimentos, visite el sitio web del Departamento de Agricultura de los Estados Unidos (USDA) en http://www. nal.usda.gov/fnic/foodcomp/Data/SR17/wtrank/sr17a305. pdf. Éste sitio proporciona un índice extenso de todos los tipos de alimentos y sus respectivos contenidos de fósforo.

\section{References}

Aleixandre, A., and M. Miguel. 2016. "Dietary fiber and blood pressure control." Food Funct 7(4):1864-71. doi: 10.1039/c5fo00950b.

Chiavaroli, L., A. Mirrahimi, J.L. Sievenpiper, D.J. Jenkins, and P.B. Darling. 2014. "Dietary fiber effects in chronic kidney disease: a systematic review and meta-analysis of controlled feeding trials." Eur J Clin Nutr. doi: 10.1038/ ejcn.2014.237.

Dahl, W. J., and M.L. Stewart. 2015. "Position of the Academy of Nutrition and Dietetics: Health Implications of Dietary Fiber." J Acad Nutr Diet 115(11):1861-70. doi: 10.1016/j.jand.2015.09.003.

Dahl, W.J., N.C. Agro, A.M. Eliasson, K.L. Mialki, J.D. Olivera, C.T. Rusch, and C.N. Young. 2016. "Health benefits of fiber fermentation." Journal of the American College of Nutrition.

Evenepoel, P., and B.K. Meijers. 2012. "Dietary fiber and protein: nutritional therapy in chronic kidney disease and beyond." Kidney Int 81(3):227-9. doi: 10.1038/ki.2011.394.

Kelly, J.T., S.C. Palmer, S.N. Wai, M. Ruospo, J.J. Carrero, K.L. Campbell, and G.F. Strippoli. 2016. "Healthy Dietary Patterns and Risk of Mortality and ESRD in CKD: A Meta-Analysis of Cohort Studies." Clin J Am Soc Nephrol. doi: 10.2215/cjn.06190616.

Krishnamurthy, V.M., G. Wei, B.C. Baird, M. Murtaugh, M.B. Chonchol, K.L. Raphael, T. Greene, and S. Beddhu. 2012. "High dietary fiber intake is associated with decreased inflammation and all-cause mortality in patients with chronic kidney disease." Kidney Int 81(3):300-6. doi: 10.1038/ki.2011.355.

Lee C.L., T.C. Li, S.Y. Lin, J.S. Wang, I.T. Lee, L.N. Tseng, Y.M. Song, S.F. Tsai, and W.H. Sheu. 2013. "Dynamic and dual effects of glycated hemoglobin on estimated glomerular filtration rate in type 2 diabetic outpatients." Am J Nephrol. 38:19-26. 
Post, R.E., A.G. Mainous, D.E. King, and K.N. Simpson. 2012. "Dietary fiber for the treatment of type 2 diabetes mellitus: a meta-analysis." J Am Board Fam Med 25(1):1623. doi: 10.3122/jabfm.2012.01.110148.

Salmean, Y.A., G.A. Zello, and W.J. Dahl. 2013. "Foods with added fiber improve stool frequency in individuals with chronic kidney disease with no impact on appetite or overall quality of life." BMC Res Notes 6:510. doi:

10.1186/1756-0500-6-510.

Shurraw, S., B. Hemmelgarn, M. Lin, S.R. Majumdar, S. Klarenbach, B. Manns, A. Bello, M. James, T.C. Turin, and M. Tonelli. 2011. "Association between glycemic control and adverse outcomes in people with diabetes mellitus and chronic kidney disease: a population-based cohort study." Arch Intern Med. 171:1920-1927.

Trumbo, P., S. Schlicker, A.A. Yates, and M. Poos. 2002. "Dietary reference intakes for energy, carbohydrate, fiber, fat, fatty acids, cholesterol, protein and amino acids." J Am Diet Assoc. 102(11):1621-30. 
Table 1. Contenido de fibra y nutrientes de algunos alimentos integrales. (USDA 2017)

\begin{tabular}{|c|c|c|c|c|c|}
\hline Cereales & Tamaño de la porción & Fibra (g) & Potasio (mg) & Fósforo (mg) & Sodio (mg) \\
\hline Health Valley Fiber 7 Flakes & $3 / 4$ taza & 4.4 & 149 & 101 & 62 \\
\hline Health Valley Oat Bran Flakes & $3 / 4$ taza & 3.0 & 128 & 129 & 142 \\
\hline Kashi 7 Whole Grain Flakes & $3 / 4$ taza & 4.4 & 92 & 65 & 109 \\
\hline Kellogg's Mueslix & $3 / 4$ taza & 4.7 & 199 & 139 & 147 \\
\hline Kellogg's All-bran Bran Buds & $1 / 4$ taza & 9.6 & 183 & 113 & 154 \\
\hline Kellogg's Miniwheats unfrosted & 15 bizcochos & 3.8 & 127 & 129 & 1 \\
\hline Post Shredded Wheat n'Bran & $3 / 4$ taza & 5.2 & 138 & 148 & 0 \\
\hline Post $^{\circledast}$ Bran Flakes & $3 / 4$ taza & 5.5 & 160 & 135 & 162 \\
\hline Post $^{\oplus}$ Grape Nuts & $1 / 4$ taza & 3.8 & 116 & 134 & 135 \\
\hline Post ${ }^{\oplus}$ Shredded Wheat n'Bran & $3 / 4$ taza & 5.2 & 138 & 148 & 0 \\
\hline Uncle Sam ${ }^{\circledast}$ Cereal & $3 / 4$ taza & 11.2 & 245 & 206 & 113 \\
\hline Weetabix Whole Grain & $3 / 4$ taza & 4.9 & 183 & 74 & 159 \\
\hline Panes & $\begin{array}{c}\text { Tamaño de la } \\
\text { porción* }\end{array}$ & Fibra (g) & Potasio (mg) & Fósforo (mg) & Sodio (mg) \\
\hline Trigo partido & 1 rebanada & 1.6 & 50 & 43 & 153 \\
\hline Avena & 1 rebanada & 1.1 & 46 & 36 & 127 \\
\hline Salvado de avena & 1 rebanada & 1.3 & 42 & 40 & 100 \\
\hline Pan integral de centeno & 1 rebanada & 1.8 & 59 & 50 & 169 \\
\hline Salvado de arroz & 1 rebanada & 1.4 & 61 & 50 & 76 \\
\hline Trigo germinado & 1 rebanada & 1.5 & 55 & 49 & 133 \\
\hline Trigo blanco & 1 rebanada & 2.6 & 36 & 29 & 134 \\
\hline Trigo integral & 1 rebanada & 1.7 & 72 & 60 & 128 \\
\hline Galletas & Tamaño de la porción & Fibra (g) & Potasio (mg) & Fósforo (mg) & Sodio (mg) \\
\hline Triscuit $^{\circledR}$ Crackers Hint of Salt & 6 galletas & 3 & $120 \mathrm{mg}$ & $10 \%$ & $50 \mathrm{mg}$ \\
\hline Ryvita ${ }^{\circledast}$ Original Crispbread & 2 rebanadas & 3 & $\mathrm{~N} / \mathrm{A}$ & $\mathrm{N} / \mathrm{A}$ & $60 \mathrm{~g}$ \\
\hline
\end{tabular}

Table 2. Contenido de fibra y nutrientes de algunos alimentos a base de granos con fibra añadida. (USDA 2017)

\begin{tabular}{|c|c|c|c|c|c|}
\hline $\begin{array}{c}\text { Alimentos a base de granos con fibra } \\
\text { añadida }\end{array}$ & Tamaño de la porción & Fibra (g) & Potasio (mg) & Fósforo (mg) & Sodio (mg) \\
\hline Arnold Sandwich Thins ${ }^{\oplus}$ & 1 rollo (43 g) & $5 \mathrm{~g}$ & $\mathrm{~N} / \mathrm{A}$ & $\mathrm{N} / \mathrm{A}$ & $170 \mathrm{mg}$ \\
\hline Barilla Pasta Added Fiber & $2 \mathrm{oz}$ & $6 \mathrm{~g}$ & $\mathrm{~N} / \mathrm{A}$ & $\mathrm{N} / \mathrm{A}$ & $0 \mathrm{mg}$ \\
\hline Carb Balance Flour Tortillas & $1(42 \mathrm{~g})$ & $13 \mathrm{~g}$ & $\mathrm{~N} / \mathrm{A}$ & $\mathrm{N} / \mathrm{A}$ & $280 \mathrm{mg}$ \\
\hline FiberOne ${ }^{\circledast}$ with Bran cereal & $1 / 2$ taza & $13.9 \mathrm{~g}$ & $112 \mathrm{mg}$ & $60 \mathrm{mg}$ & $107 \mathrm{mg}$ \\
\hline FiberOne $^{\circledast}$ Italian Bread & 1 rebanada & $3 \mathrm{~g}$ & $\mathrm{~N} / \mathrm{A}$ & $\mathrm{N} / \mathrm{A}$ & $130 \mathrm{mg}$ \\
\hline High Fiber Egg Noodles & $2 \mathrm{oz}$ & $7 \mathrm{~g}$ & $\mathrm{~N} / \mathrm{A}$ & $\mathrm{N} / \mathrm{A}$ & $10 \mathrm{mg}$ \\
\hline MM Mania Fiber Biscotti & $1 \mathrm{oz}$ & $6 \mathrm{~g}$ & $\mathrm{~N} / \mathrm{A}$ & $\mathrm{N} / \mathrm{A}$ & $70 \mathrm{mg}$ \\
\hline MM Mania Fiber Breadsticks & $1 \mathrm{oz}$ & $11 \mathrm{~g}$ & $\mathrm{~N} / \mathrm{A}$ & $\mathrm{N} / \mathrm{A}$ & $140 \mathrm{mg}$ \\
\hline Quaker Instant Oatmeal High Fiber & 1 paquete & $10 \mathrm{~g}$ & $115 \mathrm{mg}$ & $138 \mathrm{mg}$ & $213 \mathrm{mg}$ \\
\hline Wheatabix Crispy Flakes and Fiber Cereal & 1 cup & $4.4 \mathrm{~g}$ & $124 \mathrm{mg}$ & $\mathrm{N} / \mathrm{A}$ & $128 \mathrm{mg}$ \\
\hline
\end{tabular}


Table 3. Contenido de fibra y nutrientes de algunas frutas y verduras con bajo contenido de potasio.* (USDA 2017)

\begin{tabular}{|c|c|c|c|c|c|}
\hline Vegetales & Tamaño de la porción & Fibra (g) & Potasio (mg) & Fósforo (mg) & Sodio (mg) \\
\hline Brócoli (crudo, picado) & $1 / 2$ taza & 1.2 & 144 & 30 & 15 \\
\hline Repollo (crudo, picado) & $1 / 2$ taza & 1.1 & 76 & 12 & 8 \\
\hline Zanahoria (cruda) & 1 pequeña & 1.4 & 160 & 18 & 34 \\
\hline Coliflor (crudo, picado) & $1 / 2$ taza & 1.1 & 160 & 24 & 16 \\
\hline Apio (tallo, crudo) & 1 mediano & 0.6 & 104 & 10 & 32 \\
\hline Maíz (granos, cocinados) & $1 / 2$ taza & 2.1 & 198 & 72 & 2 \\
\hline Pepino (crudo, pelado, picado,) & $1 / 2$ taza & 0.5 & 90 & 14 & 1 \\
\hline Berenjena (pelada, en cubos, cocinada) & $1 / 2$ taza & 1.2 & 61 & 7 & 0 \\
\hline Ejotes (cocinados) & $1 / 2$ taza & 2.0 & 91 & 18 & 1 \\
\hline Pimiento verde (picado, crudo) & $1 / 2$ taza & 1.3 & 130 & 15 & 2 \\
\hline Rábano (crudo, en rodajas) & $1 / 2$ taza & 0.9 & 135 & 12 & 23 \\
\hline Calabacín (crudo, con cáscara, picado) & $1 / 2$ taza & 0.6 & 162 & 24 & 5 \\
\hline Fruta & Tamaño de la porción & Fibra (g) & Potasio (mg) & Fósforo (mg) & Sodio (mg) \\
\hline Manzana & 1 pequeña & 3.6 & 159 & 16 & 1 \\
\hline Arándanos & $1 / 2$ taza & 1.8 & 57 & 9 & 1 \\
\hline Moras & $1 / 2$ taza & 3.8 & 117 & 16 & 1 \\
\hline Cerezas (dulces, con tallo) & $1 / 2$ taza & 1.4 & 153 & 14 & 0 \\
\hline Toronja (cruda, rosada y roja) & $1 / 2$ entera & 1.4 & 156 & 11 & 0 \\
\hline Uvas (rojas o verdes) & 15 & 0.7 & 140 & 15 & 1 \\
\hline Pera con cáscara & 1 pequeña & 4.6 & 172 & 18 & 1 \\
\hline Piña (trozos) & $1 / 2$ taza & 1.2 & 90 & 7 & 1 \\
\hline Ciruela con cáscara & 1 mediana & 0.9 & 104 & 11 & 0 \\
\hline Frambuesas & $1 / 2$ taza & 4.0 & 93 & 18 & 1 \\
\hline Mandarina & 1 pequeña & 1.4 & 126 & 15 & 2 \\
\hline Fresas (enteras) & $1 / 2$ taza & 1.4 & 110 & 17 & 1 \\
\hline
\end{tabular}

